\title{
The Microbiome Studies in Metabolic Diseases have Advanced but are Poorly Standardized and Lack a Mechanistic Perspective
}

\author{
Satu Pekkala ${ }^{1 *}$, Eveliina Munukka², Anniina Rintala ${ }^{2}$ and Pentti Huovinen ${ }^{2,3}$
}

${ }^{1}$ Department of Health Sciences, University of Jyväskylä, Jyväskylä, Finland

${ }^{2}$ Department of Medical Microbiology and Immunology, University of Turku, Turku, Finland

${ }^{3}$ Division of Health Protection, National Institute of Health and Welfare, Turku, Finland

During the last decades hundreds of studies have reported the association of Gut Microbiota (GM) with obesity and related metabolic disorders [1]. However, recently the microbiome studies were criticized about the lack of skepticism [2]. The author of the article questioned the role of GM in different diseases and asked whether the detected differences between the subjects biologically matter? We do believe that the role of microbiota in metabolic diseases is true. The impact of GM on human health should not be surprising since our gut shapes up the symbiotic habitat for at least 100 trillion microbial cells that count up to 100 -fold more genes than our genome [1].

However, we consider that the current problem in microbiome studies is the lack of standardization, which disables the reproducibility of the results. In our experience, the different sample storage and DNA extraction methods cause variation in the amount of DNA and in the abundance of certain bacterial groups (Rintala et al., in preparation). In population studies genders and different age groups are being continuously mixed, which is incorrect since for instance the inflammatory mechanisms and association of GM with metabolic parameters vary between genders [3], and moreover, GM composition changes during aging [4]. Further, $16 \mathrm{~S}$ sequencing yields thousands of OTUs, whose identification varies according to the database that is being used. This may create significant bias knowing the geographical differences in human GM [5]. Moreover, some of the sequencing results have not been reproduced using other methods, such as HITChip [6]. As it is required for other high-throughput analyses, i.e. Microarray, the $16 \mathrm{~S}$ sequencing results should be validated using another conventional method, such as flow cytometry combined with FISH or with real-time quantitative PCR (qPCR). When qPCR was accepted as a standard method to quantify gene expression levels, The Minimum Information for Publication of Quantitative Real-Time PCR Experiments (MIQE) guidelines were established to promote consistency between laboratories. The same should be done to standardize the GM analyses.

Another question that one should ask is that how many associative studies do we still need to show that GM plays a role in different diseases? We believe that the era of associative studies on GM should now have reached its' end. The research must take one step forward and use the information gained from the existing studies to test the causative roles and underlying mechanisms of the microbes in different diseases.

An associative study can never identify the underlying mechanisms of a disease. Therefore the high-throughput sequencing and metagenome analyses should serve as a rationale to create animal models for validating the fundamental roles of the GM. If a specific bacterial species is associated with obesity, its' causal role in obesity should be confirmed by colonizing an animal with that species. A great effort in unraveling the underlying mechanisms that connect the microbes to certain diseases has been made by Drs Cani and Delzenne [7] and by the laboratory of Dr. Gewirtz [8]. However, setting up controlled animal experiments for such mechanistic studies lead to another question: how much do animal experiments reflect reality? Most of the microbiome studies are made in germ-free or antibiotictreated mice lacking the gut microbial community. These situations in human gut likely never exist, and therefore the observations obtained in microbe-free mice cannot be extended to humans. Other models, such as humanized mice should be considered for the experimental studies.

Finally, one important factor, which too often is ignored in GM studies, is the diet. The GM is readily influenced by diet [9], and thus their association with metabolic diseases may depend on the dietary composition. Many interventional human studies have failed to modify the GM composition in obese subjects possibly due to inter-individual variation of the initial composition before the dietary intervention [10]. Here, the metagenome analyses should identify the metabolic capacities of the enriched bacterial species in different diseases and conditions. This knowledge should be further applied to animal models and dietary interventions to elucidate whether and how specific dietary choices through modifying the abundance of GM can be used to treat specific diseases.

\section{References}

1. Karlsson F, Tremaroli V, Nielsen J, Bäckhed F (2013) Assessing the human gut microbiota in metabolic diseases. Diabetes 62: 3341-3349.

2. Hanage WP (2014) Microbiology: Microbiome science needs a healthy dose of scepticism. Nature 512: 247-248.

3. Feng J, Tang H, Li M, Pang X, Wang L, et al. (2014) The abundance of fecal Faecalibacterium prausnitzii in relation to obesity and gender in Chinese adults. Arch Microbiol 196: 73-77.

4. Claesson MJ, Jeffery IB, Conde S, Power SE, O'Connor EM, et al. (2012) Gut microbiota composition correlates with diet and health in the elderly. Nature 488: $178-184$

5. Suzuki TA, Worobey M (2014) Geographical variation of human gut microbial composition. Biol Lett 10: 20131037.

6. Biagi E, Nylund L, Candela M, Ostan R, Bucci L, et al. (2012) Through ageing, and beyond: gut microbiota and inflammatory status in seniors and centenarians. PLoS One 5: e10667.

*Corresponding author: Satu Pekkala, Department of Health Sciences, University of Jyväskylä, PO Box 35, FI-40014 University of Jyväskylä, Finland, Tel.:+358 45 35828 98; Fax: +358 14 2602011; E-mail: satu.p.pekkala@jyu.fi

Received October 27, 2014; Accepted December 13, 2014; Published Decembe 15,2014

Citation: Pekkala S, Munukka E, Rintala A, Huovinen P (2014) The Microbiome Studies in Metabolic Diseases have Advanced but are Poorly Standardized and Lack a Mechanistic Perspective. J Diabetes Metab 6: 480. doi:10.4172/21556156.1000480

Copyright: ( 2014 Pekkala S, et al. This is an open-access article distributed unde the terms of the Creative Commons Attribution License, which permits unrestricted use, distribution, and reproduction in any medium, provided the original author and source are credited. 
Citation: Pekkala S, Munukka E, Rintala A, Huovinen P (2014) The Microbiome Studies in Metabolic Diseases have Advanced but are Poorly Standardized and Lack a Mechanistic Perspective. J Diabetes Metab 6: 480. doi:10.4172/2155-6156.1000480

Page 2 of 2

7. Cani PD, Delzenne NM (2009) The role of the gut microbiota in energy metabolism and metabolic disease. Curr Pharm Des 15: 1546-1558.

8. Carvalho FA, Aitken JD, Vijay-Kumar M, Gewirtz AT (2012) Toll-like receptorgut microbiota interactions: perturb at your own risk! Annu Rev Physiol 74 177-198
9. Sekirov I, Russell SL, Antunes LC, Finlay BB (2010) Gut microbiota in health and disease. Physiol Rev 90: 859-904.

10. Flint HJ, Scott KP, Louis P, Duncan SH (2012) The role of the gut microbiota in nutrition and health. Nat Rev Gastroenterol Hepatol 9: 577-589. 